DEMONSTRATIO MATHEMATICA

Vol. XXXIV No $4 \quad 2001$

\title{
Aldona Szukała
}

\section{ON THE APPLICATION OF SOME MEASURES OF NONCOMPACTNESS TO EXISTENCE THEOREMS FOR AN $m$ TH ORDER DIFFERENTIAL EQUATION}

Assume that $I=[0, a], E$ is a Banach space, $B=\{x \in E:\|x\| \leq b\}$ and $f: I \times B \mapsto E$ is a bounded uniformly continuous function.

In this paper we shall give sufficient conditions for the existence of solutions of the Cauchy problem

$$
\begin{gathered}
x^{(m)}=f(t, x) \\
x(0)=0, x^{\prime}(0)=\eta_{1}, \ldots, x^{(m-1)}(0)=\eta_{m-1},
\end{gathered}
$$

where $\eta_{1}, \ldots, \eta_{m-1} \in E$.

These conditions are expressed in terms of axiomatic measures of noncompactness introduced by Banaś and Goebel [2]. Our considerations are connected with the paper [5]. In contrast to [5], we shall use in our proof some ideas from papers [3] and [1].

Analogously, as in [2] and [1], let $M_{E}$ denote the family of all nonempty and bounded subsets of $E$ and $N_{E}$ the family of all nonempty and relatively compact sets in $E$.

Recall that a function $\mu: M_{E} \mapsto[0, \infty)$ is said to be a measure of noncompactness in $E$ if it satisfies the following conditions:

$1^{\circ}$ the family $\operatorname{ker} \mu=\left\{X \in M_{E}: \mu(X)=0\right\}$ is nonempty and $\operatorname{ker} \mu \in$ $N_{E}$

$2^{\circ}$ if $X \subset Y$, then $\mu(X) \leq \mu(Y)$,

$3^{\circ} \mu(\bar{X})=\mu(X)$,

$4^{\circ} \mu(\operatorname{convX})=\mu(\mathrm{X})$,

$5^{\circ} \mu(\lambda \cdot X+(1-\lambda) Y)=\lambda \cdot \mu(X)+(1-\lambda) \mu(Y)$ for $\lambda \in[0,1]$,

$6^{\circ}$ if $X_{n} \in M_{E}, X_{n}=\bar{X}_{n}$ and $X_{n+1} \subset X_{n}$ for $n=1,2, \ldots$ and $\lim _{n \rightarrow \infty} \mu\left(X_{n}\right)=0$, then $X_{\infty}=\bigcap_{n=1}^{\infty} X_{n} \neq \emptyset$. 
Let $\mu$ be a measure of noncompactness in the above sense. Moreover, we assume

$7^{\circ} \mu(\{a\})=0$ for all $a \in E$,

$8^{\circ} \mu(a+X)=\mu(X)$ for $a \in E$ and $X \in M_{E}$.

Then, by $5^{\circ}$ we have

$9^{\circ} \mu(\lambda X) \leq \lambda \mu(X)$ for $0 \leq \lambda \leq 1$.

Set $E_{\mu}=\{x \in E:\{x\} \in$ ker $\mu\}$.

Denote by $B(0, r)$ the ball with center 0 and radius $r$.

The main result of the paper is the following

THEOREM. Suppose that there is a continuous function $u:[0, a] \mapsto \mathbb{R}_{+}$such that $u(t)>0$ for $t>0, u(0)=\ldots u^{(m-1)}(0)=0$ and $u^{(m)}(t)>0$ is Lebesque intergrable. If

$$
\mu(f(t, X)) \leq \frac{u^{(m)}(t)}{u(t)} \mu(X) \text { for } X \subset B \text { and } t \in(0, a)
$$

and

$$
\lim _{\substack{t \rightarrow 0^{+} \\ r \rightarrow 0^{+}}} \frac{\mu(f(t, B(0, r)))}{u^{(m)}(t)}=0,
$$

then there exists an interval $J=[0, d]$ such that the problem (1)-(2) has at least one solution defined on $J$.

Proof. Put $M=\sup \{\|f(t, x)\|: t \in I, x \in B\}$. Choose a positive number $d$ such that $d \leq a$ and

$$
\sum_{j=1}^{m-1}\left\|\eta_{j}\right\| \frac{d^{j}}{j !}+M \frac{d^{m}}{m !} \leq b
$$

Let $C=C(J, E)$ be the Banach space of continuous functions $J \mapsto E$ and let $\tilde{B} \subset C$ be the subset of those functions with values in $B$.

We define a mapping $F$ by

$$
F(x)(t)=p(t)+\frac{1}{(m-1) !} \int_{0}^{t}(t-s)^{m-1} f(s, x(s)) d s \quad(t \in J, x \in \tilde{B}),
$$

where $p(t)=\sum_{j=1}^{m-1} \eta_{j} \frac{t^{j}}{j !}$. It is known $(c f .[4])$ that $F$ is a continuous mapping $\tilde{B} \mapsto \tilde{B}$ and a function $x \in C$ is a solution of (1)-(2) iff $x=F(x)$. Moreover, by the Lagrange theorem, we have

$$
\|F(x)(t)-F(x)(\tau)\| \leq K|t-\tau| \text { for } x \in \tilde{B}, \quad t, \tau \in J
$$


where $K=\sum_{j=1}^{m-1}\left\|\eta_{j}\right\| \frac{d^{j-1}}{(j-1) !}+M \frac{d^{m-1}}{(m-1) !}$.

Denote by $X_{o}$ the set of all functions $x \in C(J, E)$ such that $x(0)=0$ and $\|x(t)-x(\tau)\| \leq K|t-\tau|$ for $t, \tau \in J$. The set $X_{o}$ is nonempty, bounded, closed, convex and equicontinuous.

Set $X_{n+1}=\overline{c o n v} F\left(X_{n}\right)(n=0,1, \ldots)$. The mapping $F$ is continuous and maps $X_{o}$ into itself. Since the set $X_{o}$ is convex and closed,

$$
X_{1}=\overline{\operatorname{conv}} F\left(X_{o}\right) \subset \overline{\operatorname{conv}} X_{o}=X_{o} .
$$

Similarly $X_{n+1} \subset X_{n}$. Let $X_{n}(t)=\left\{x(t): x \in X_{n}\right\}$. As $X_{n+1} \subset X_{n}$, we have $X_{n+1}(t) \subset X_{n}(t)$. Hence $\mu\left(X_{n+1}(t)\right) \leq \mu\left(X_{n}(t)\right)$.

Putting $v_{n}(t)=\mu(X(t))$, we get $0 \leq v_{n+1}(t) \leq v_{n}(t)(n=0,1, \ldots)$ and all these functions are equicontinuous. Therefore the sequence $v_{n}(t)$ converges uniformly to a function $v_{\infty}(t)=\lim _{n \rightarrow \infty} v_{n}(t)$.

First we assume that $d \leq 1$.

By (4) for given $\varepsilon>0$ there exists $\delta>0$ such that

$$
\mu(f(t, B(0, K t))) \leq \varepsilon u^{(m)}(t) \text { for } 0 \leq t \leq \delta .
$$

Since $f$ is uniformly continuous and $X_{n}$ is equicontinuous, it follows that for given $t>0$ the set of functions

$$
s \mapsto(t-s)^{m-1} f(s, x(s))^{\circ} \text { for } x \in X_{n}
$$

is equicontinuous on $[0, t]$. For $x \in X_{\circ}$ we have $\|x(t)\| \leq K t$. Fix $t \in(0, \delta]$.

Applying $7^{\circ}, 8^{\circ}$ and Lemma 7.2.4 of [1] we obtain

$$
\begin{aligned}
v_{n+1}(t) & =\mu\left(X_{n+1}(t)\right)=\mu\left(\overline{\operatorname{conv}} F\left(X_{n}\right)(t)\right)=\mu\left(F\left(X_{n}\right)(t)\right) \\
& =\mu\left(\left\{p(t)+\frac{1}{(m-1) !} \int_{0}^{t}(t-s)^{m-1} f(s, x(s)) d s: x \in X_{n}\right\}\right) \\
& =\mu\left(\left\{\frac{1}{(m-1) !} \int_{0}^{t}(t-s)^{m-1} f(s, x(s)) d s: x \in X_{n}\right\}\right) \\
& \leq \int_{0}^{t} \mu\left(\left\{\frac{1}{(m-1) !}(t-s)^{m-1} f(s, x(s)): x \in X_{n}\right\}\right) d s \\
& \leq \int_{0}^{t} \frac{1}{(m-1) !}(t-s)^{m-1} \mu\left(\left\{f(s, x(s)): x \in X_{n}\right\}\right) d s .
\end{aligned}
$$

The last inequality follows from $9^{\circ}$, because

$$
\frac{(t-s)^{m-1}}{(m-1) !} \leq 1
$$


As $X_{n} \subset X_{o}$, we have $\|x(t)\| \leq K t$ for $x \in X_{n}$. In view of (6) this implies that

$$
v_{n+1}(t) \leq \int_{0}^{t} \frac{1}{(m-1) !}(t-s)^{m-1} \varepsilon u^{(m)}(s) d s=\varepsilon u(t),
$$

so that

$$
v_{n+1}(t)=\mu\left(X_{n+1}(t)\right) \leq \varepsilon u(t) \text { for } t \in[0, \delta) \text { and } n=0,1, \ldots
$$

Thus $v_{\infty}(t)=\mu\left(X_{\infty}(t)\right) \leq \varepsilon u(t)$ for $t \in(0, \delta]$. Fix $t \in(0, d)$.

Similar arguments show that

$$
\begin{aligned}
v_{n+1}(t) & =\mu\left(X_{n+1}(t)\right)=\mu\left(\overline{\operatorname{conv}} F\left(X_{n}\right)(t)\right)=\mu\left(F\left(X_{n}\right)(t)\right) \\
& =\mu\left(\left\{p(t)+\frac{1}{(m-1) !} \int_{0}^{t}(t-s)^{m-1} f(s, x(s)) d s: x \in X_{n}\right\}\right) \\
& =\mu\left(\left\{\frac{1}{(m-1) !} \int_{0}^{t}(t-s)^{m-1} f(s, x(s)) d s: x \in X_{n}\right\}\right) \\
& \leq \int_{0}^{t} \mu\left(\left\{\frac{1}{(m-1) !}(t-s)^{m-1} f(s, x(s)): x \in X_{n}\right\}\right) d s \\
& \leq \int_{0}^{t} \frac{1}{(m-1) !}(t-s)^{m-1} \mu\left(\left\{f(s, x(s)): x \in X_{n}\right\}\right) d s \\
& \leq \int_{0}^{t} \frac{(t-s)^{m-1}}{(m-1) !} \frac{u^{(m)}(s)}{u(s)} \mu\left(X_{n}(s)\right) d s .
\end{aligned}
$$

From (7) it is clear that there exists the integral

$$
\int_{0}^{t} \frac{(t-s)^{m-1}}{(m-1) !} \frac{u^{(m)}(s)}{u(s)} \mu\left(X_{n}(s)\right) d s .
$$

Thus

$$
v_{n+1}(t) \leq \int_{0}^{t} \frac{(t-s)^{m-1}}{(m-1) !} \frac{u^{(m)}(s)}{u(s)} v_{n}(s) d s .
$$

By the Lebesque dominated convergence theorem, we get

$$
v_{\infty}(t) \leq \int_{0}^{t} \frac{(t-s)^{m-1}}{(m-1) !} \frac{u^{(m)}(s)}{u(s)} v_{\infty}(s) d s \quad \text { dla } t \in(0, d) .
$$

Put $z(t)=\frac{v_{\infty}(t)}{u(t)}$ for $t \in(0, d)$ and $z(0)=0$. By (7) the function $z$ is continuous. Suppose that $z$ is not identically zero on $[0, d)$. Let $\tau$ be the 
smallest real number such that $z(\tau)=\max _{t \in J} z(t)$. Then $0 \leq z(s)<z(\tau)$ for $s \in[0, \tau)$. By (8) we have

$$
\begin{aligned}
z(\tau) & =\frac{v_{\infty}(\tau)}{u(\tau)} \leq \frac{1}{u(\tau)(m-1) !} \int_{0}^{\tau}(\tau-s)^{m-1} \frac{u^{(m)}(s)}{u(s)} v_{\infty}(s) d s \\
& =\frac{1}{u(\tau)(m-1) !} \int_{0}^{\tau}(\tau-s)^{m-1} u^{(m)}(s) z(s) d s \\
& <\frac{z(\tau)}{u(\tau)} \frac{1}{(m-1) !} \int_{0}^{\tau}(\tau-s)^{m-1} u^{(m)}(s) d s=\frac{z(\tau)}{u(\tau)} \cdot u(\tau)=z(\tau),
\end{aligned}
$$

a contradiction. Thus $z(t) \equiv 0$ on $[0, d]$, i.e. $v_{\infty}(t)=0$ on $[0, d]$.

Set $\mu_{C}(X)=\max \{\mu(X(t)): t \in J\}$ for $X$ equicontinuous. Then $\mu_{C}$ is a measure of noncompactness (cf. [2], Theorem 11.2, p.59). Since

$$
\lim _{n \rightarrow \infty} \max \left\{v_{n}(t): t \in J\right\}=0,
$$

$\mu_{C}\left(X_{n}\right) \rightarrow 0$ as $n \rightarrow \infty$. Hence the set $X_{\infty}=\bigcap_{n=1}^{\infty} X_{n}$ is nonempty, convex, closed and $X_{\infty} \subset E_{\mu}$.

Now we can apply the Schauder fixed point theorem to the mapping $F \mid X_{\infty}$, which yields the existence of $x \in X_{\infty}$ such that $x=F(x)$, i.e. $x$ is a solution of $(1)-(2)$.

Suppose now $d>1$. For $t \in[1, d]$ the function $f(t, x)$ satisfies the $\mu$ Lipschitz condition with the integrable function $\lambda(t)=\frac{u^{(m)}(t)}{u(t)}$, i.e.

$$
\mu(f(t, X)) \leq \lambda(t) \mu(X) \text { for } t \in[1, d] .
$$

The equation $x^{(m)}=f(t, x)$ is equivalent to the first order system. Applying now Theorem 7.3.1 of [1], we see that the solution can be continued to the interval $[0, h], 1<h \leq d$. If $h<d$, then

$$
\|x(t)\| \leq \sum_{j=1}^{m-1}\left\|\eta_{j}\right\| \frac{h^{j}}{j !}+M \frac{h^{m}}{m !}<b \quad \text { for } t \in[0, h],
$$

so that $x(h) \in \operatorname{Int} B$. Thus the solution $x(t)$ can be continued to the interval $J=[0, d]$.

REMARK. Assume that $A:(0, d] \mapsto \mathbb{R}$ is a function such that $A^{\prime}(t)=a(t)$ for almost every $t \in(0, d], \lim _{t \rightarrow 0+} A(t)=-\infty$, where $a(t) \geq 0$ for $(0, d]$. If we take $m=1$ and $u(t)=\exp A(t)$ in our theorem, we obtain Theorem 7.4.2 from [1]. 
The following functions will be useful as some examples

1) $a(t)=\frac{c}{t}(c>1), A(t)=c \ln t, t \in(0,1]$,

2) $a(t)=\frac{1}{t^{2}}, A(t)=-\frac{1}{t}, t \in(0,1]$.

\section{References}

[1] J. Ban aś, Applications of measures of noncompactness to various problems, Zeszyty Nauk. Politech: Rzeszowskiej 34 (1987).

[2] J. Banaś, K. Goebel, Measures of Noncompactness in Banach Spaces, Marcel Dekker, New York-Basel, 1980.

[3] K. Goebel, W. Rzymowski, An existence theorem for the equation $x^{\prime}=f(t, x)$ in Banach space, Bull. Acad. Pol. Sci. Math. 18 (1970), 367-370.

[4] S. Szufla, On Volterra integral equations in Banach spaces, Funkcialaj Ekvac. 20 (1977), 247-258.

[5] S. Szufla, A. Szukala, An existence theorem for the equation $x^{m}=f(t, x)$ in Banach spaces, Funct. Appr. 25 (1997), 181-188.

FACULTY OF MATHEMATICS AND COMPUTER SCIENCE

A. MICKIEWICZ UNIVERSITY

J. Matejki $48 / 49$

60-769 POZNAŃ, POLAND

Received December 14, 2000. 